\title{
Self-activation of Serine/Threonine Kinase AfsK on Autophosphorylation at Threonine-168
}

\author{
Ayami Tomono, Mari Mashiko, Tadahiro Shimazu, Hirotaka Inoue, Hiromichi Nagasawa, \\ Minoru Yoshida, Yasuo Ohnishi, Sueharu Horinouchi
}

Received: November 29, 2005 / Accepted: February 2, 2006

(C) Japan Antibiotics Research Association

\begin{abstract}
A Hanks-type protein kinase AfsK autophosphorylates on threonine residue(s) and phosphorylates AfsR, a global regulator for secondary metabolism in Streptomyces coelicolor A3(2). Mass spectrometry of a tryptic digest of the autophosphorylated form of $\mathrm{AfsK} \Delta \mathrm{C}$ corresponding to the kinase catalytic domain (Met-1 to Arg-311) of AfsK, together with subsequent site-directed mutagenesis of the candidate amino acids, identified threonine-168 as a single autophosphorylation site. Threonine-168 is located in the activation loop that is known for some Ser/Thr kinases to modulate kinase activity on phosphorylation of one or more threonine residues within the loop. Consistent with this, mutant T168D, in which Thr-168 was replaced by Asp, became a constitutively active kinase; it phosphorylated AfsR to the same extent as AfsK $\Delta \mathrm{C}$ produced in and purified from Escherichia coli cells during which a considerable population of it had been already phosphorylated intermolecularly. All these findings show that autophosphorylation or intermolecular phosphorylation of threonine-168 in AfsK accounts for the self-activation of its kinase activity.
\end{abstract}

Keywords serine/threonine kinase, autophosphorylation, self-activation, antibiotic production, Streptomyces
A number of proteins in Streptomyces, including Streptomyces coelicolor A3(2), are phosphorylated on their serine/threonine and tyrosine residues in response to developmental phases $[1,2]$. Recent completion of the genome projects of $S$. coelicolor A3(2) [3] and $S$. avermitilis [4] has revealed the presence of about 40 proteins having a kinase catalytic domain similar to those of the typical eukaryotic serine/threonine and tyrosine kinases. All these observations clearly show that a given Streptomyces strain possesses several protein kinases of eukaryotic type, some of which regulate growth, morphological development and secondary metabolism. Of the protein serine/threonine kinases in Streptomyces, AfsK that phosphorylates serine/threonine residues of AfsR was first discovered, representing the first instance in the bacterial world in which the ability of a bacterial Hankstype protein kinase to phosphorylate an exogenous protein has been demonstrated [5]. The AfsK-AfsR system in S. coelicolor A3(2) globally controls the biosynthesis of secondary metabolites including actinorhodin, undecylprodigiosin, methylenomycin, and a calciumdependent antibiotic [2].

Autophosphorylated amino acid residues of Hanks kinases in prokaryotes, such as PrkC in Bacillus subtilis [6], PknB in Mycobacterium tuberculosis [7 9], PknD, PknE and PknF in M. tuberculosis [9], and $\mathrm{PknH}$ in $M$.
S. Horinouchi (Corresponding author), A. Tomono, M. Mashiko, T. Shimazu, M. Yoshida, Y. Ohnishi: Department of Biotechnology, Graduate School of Agriculture and Life Sciences, University of Tokyo, Bunkyo-ku, Tokyo 113-8657, Japan,

E-mail: asuhori@mail.ecc.u-tokyo.ac.jp
T. Shimazu, M. Yoshida: Chemical Genetics Laboratory, RIKEN, Wako-shi, Saitama 351-0198, Japan

H. Inoue, H. Nagasawa: Department of Applied Biological Chemistry, Graduate School of Agriculture and Life Sciences, University of Tokyo, Bunkyo-ku, Tokyo 113-8657, Japan 
tuberculosis [10], have been determined. All these kinases autophosphorylate on two or more threonine residues in the activation loop. The two phosphorylated threonines in PknB are both important for activation of its kinase activity $[8,9]$. On the other hand, phosphorylation of one threonine residue in the activation loop is important for mammalian cAMP-dependent protein kinase A (PKA) $[11,12]$ and AMP-activated protein kinase (AMPK) [13]. Because of high similarity of AfsK in amino acid sequence and accordingly in tertiary structure to these kinases, we expected that AfsK would also autophosphorylate one or more threonine residues in the activation loop. In the present study, we determined the amino acid residue(s) that AfsK phosphorylates intermolecularly. AfsK was found to autophosphorylate on a single threonine residue at position 168 and to enhance its kinase activity toward AfsR as a substrate. In addition, amino acid replacement of Thr-168 by aspartate yielded a constitutively active AfsK kinase, as is found for PKA and AMPK.

\section{Purification of AfsK $\Delta C$ and AfsR in Active Forms}

AfsK produced as fusion proteins with thioredoxin and glutathione $S$-transferase in Escherichia coli formed inactive, inclusion bodies, despite various cultural conditions, such as cultivation at low temperatures [14]. We therefore established an efficient process for refolding AfsK in a highly active form for determination of the autophosphorylated residue(s). The AfsK protein we used for the present study was AfsK $\Delta \mathrm{C}$ (Met-1 to Arg-311 covering the kinase catalytic domain) fused to thioredoxin (TRX), which had a structure of TRX-His tag-AfsK (Met-1 to Arg-311)-His tag containing two His-tags at its N- and C-termini. The expression plasmid, pTRX-K $\Delta \mathrm{C}$-His, was constructed by standard DNA manipulation. AfsK $\Delta \mathrm{C}$ showed the same kinase activity toward AfsK itself and AfsR, as did AfsK of the full length [14]. In addition, phosphorylation patterns on AfsK and AfsR were also the same; the major phosphorylated residues of both substrates were threonine, as determined by phosphoamino acid analysis by cellulose thin-layer chromatography (TLC) [14, 15]. E. coli BL21 (DE3) pLysS harboring pTRX-K $\Delta \mathrm{C}-\mathrm{His}$ was cultured and $\mathrm{AfsK} \Delta \mathrm{C}$ was collected from an insoluble fraction, as previously described [14]. After solubilization of $\operatorname{AfsK} \Delta \mathrm{C}$ with $8 \mathrm{M}$ urea, the sample was directly applied to an FPLC column (Ni-nitrilotriacetic acid column; Qiagen) equilibrated with a buffer containing $8 \mathrm{M}$ urea and refolded during a linear gradient of 6 to $0 \mathrm{M}$ urea. AfsK $\Delta \mathrm{C}$ was then eluted by a linear gradient of $50 \sim 500 \mathrm{mM}$ imidazole. The gradual removal of urea by using a linear gradient within the column in FPLC gave $\operatorname{AfsK} \Delta \mathrm{C}$ with a much higher autophosphorylation activity than the step- wise removal of urea; only $1 \mu \mathrm{g} \operatorname{AfsK} \Delta \mathrm{C}$ yielded a phosphorylated signal on autoradiogram, whereas more than $5 \mu \mathrm{g}$ of the AfsK $\Delta \mathrm{C}$ protein, prepared through the step-wise removal of urea, was required for detection of a ${ }^{32} \mathrm{P}$-signal (data not shown). AfsR as a substrate of AfsK was purified from a soluble fraction of $E$. coli harboring pET16-afs $R$, as previously described [15]. Protein concentrations were measured with a Bio-Rad dye-binding protein assay kit using bovine serum albumin as the standard.

\section{Intermolecular Phosphorylation of AfsK $\Delta \mathrm{C}$}

A considerable population of AfsK purified from E. coli is autophosphorylated in E. coli cells and during purification (see below). For autophosphorylation of the nonphosphorylated population of $\mathrm{AfsK} \Delta \mathrm{C}$, the standard reaction mixture, containing $15 \mathrm{pmol}$ of the kinase in $10 \mathrm{mM}$ Tris- $\mathrm{HCl}$ (pH 7.2), $10 \mathrm{mM} \mathrm{MnCl}, 10 \mathrm{mM} \mathrm{MgCl}_{2}$, $0.1 \mathrm{mM}$ ATP, $10 \mu \mathrm{Ci} \quad(370 \mathrm{kBq}) \quad\left[\gamma_{-}{ }^{32} \mathrm{P}\right] \mathrm{ATP}, \quad 1 \mathrm{mM}$ dithiothreitol in a total volume of $20 \mu \mathrm{l}$, was incubated at $30^{\circ} \mathrm{C}$ for 15 minutes. The reaction was terminated by boiling for 2 minutes after adding $4 \mu \mathrm{l}$ of a dye solution. Phosphoamino acid analysis by cellulose TLC revealed the presence of phospho-threonine as an overwhelmingly large population and phospho-serine as a faint population (data not shown), as was found for full length AfsK $[14,16]$.

\section{Mass Spectrometry}

For preparation of phosphorylated $\operatorname{AfsK} \Delta \mathrm{C}$, the stopped reaction mixture was fractionated by SDS-polyacrylamide gel electrophoresis. After the gel had been stained with Coomassie brilliant blue, a band corresponding to $\mathrm{AfsK} \Delta \mathrm{C}$ was excised, and gel pieces were destained with $50 \% \mathrm{CH}_{3} \mathrm{CN}$ in $50 \mathrm{mM} \mathrm{NH} \mathrm{NHCO}_{3}$ solution. After removal of the supernatant, cysteine residues were reduced with dithiothreitol and carbamido-methylated with iodoacetamide. The proteins were then digested with trypsin at $37^{\circ} \mathrm{C}$ for overnight. The tryptic peptides were recovered by sequentially adding $50 \% \mathrm{CH}_{3} \mathrm{CN} / 1 \%$ trifluoroacetic acid (TFA), $20 \% \mathrm{HCOOH} / 25 \% \mathrm{CH}_{3} \mathrm{CN} / 15 \%$ 2-propanol, and $80 \% \mathrm{CH}_{3} \mathrm{CN}$ solutions. The supernatants were collected and pooled in one tube, and the volume was reduced under vacuum. The dried tryptic peptides were suspended in $2 \% \mathrm{CH}_{3} \mathrm{CN} / 0.1 \%$ TFA and applied to the following LC-MS/MS system. Chromatographic separation was accomplished on the MAGIC 2002 HPLC system (Michrom BioResources, Inc., Auburn, CA). Peptide samples were loaded onto a Cadenza C18 custom-packed column $(0.2 \times 50 \mathrm{~mm}$; Michrom BioResources, Inc.), in which Cadenza CD-C18 resin (Imtakt Corporation, Kyoto) was packed. Peptides were eluted using a linear gradient of 
Table 1 Tryptic peptides containing a single phosphate, as determined by LC-MS

\begin{tabular}{|c|c|c|c|}
\hline $\begin{array}{c}\text { Observed } \\
m / z\left[\mathrm{MH}_{2}^{++}\right] \\
\left(\left[\mathrm{MH}_{2}^{++}\right] \times 2-2.00\right)\end{array}$ & $\begin{array}{c}\text { Molecular weight } \\
\text { phosphorylated form } \\
\text { (non-phosphorylated form) }\end{array}$ & Delta & Sequence \\
\hline $860.58(1719.16)$ & $1718.78(1638.80)$ & 0.38 & 68-AVSGFYTAAVVDADPR-83 \\
\hline 1119.29 (2236.58) & $2236.45(2156.48)$ & 0.13 & 117-WLAAGVAEALQSIHGAGLVHR-137 \\
\hline $1095.54(2189.08)$ & $2189.42(2109.44)$ & -0.34 & 167-LTMTNVAVGTPAYMSPEQAK-186 \\
\hline
\end{tabular}

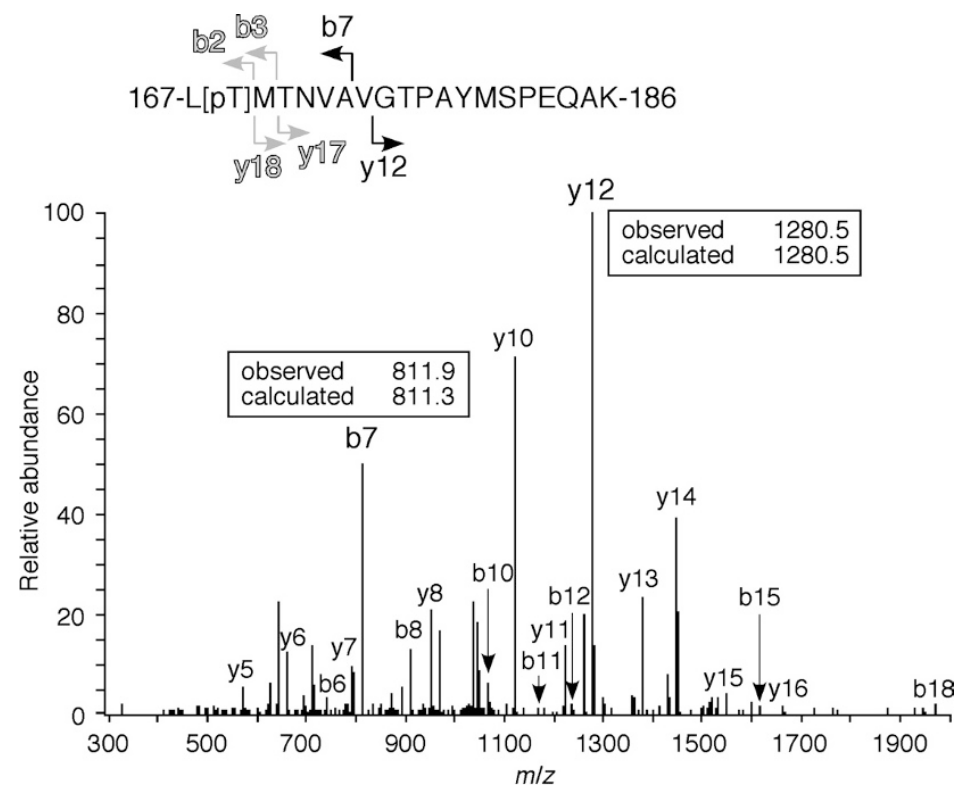

Fig. 1 Automatic nanoflow LC-MS/MS analysis of a phosphorylated peptide (positions from 167 to 186; m/z 1095.54).

The observed and calculated values of the N-terminal (b-ion series) and C-terminal (y-ion series) peptide fragment ions for b7 and y12 are highlighted, on the assumption that Thr-168 is phosphorylated. The MS/MS data show the presence of a phosphate group on Thr-168 or Thr-170. Observed values in Da: b6 (740.7), b7 (811.3), b8 (911.5), b10 (1069.5), b11 (1166.2), b12 (1236.7), b15 (1618.3), b18 (1973.5); y5 (572.4), y6 (659.6), y7 (790.5), y8 (954.5), y10 (1122.5), y11 (1223.5), y12 (1280.5), y13 (1379.5), y14 (1450.6), y15 (1549.7), y16 (1663.7).

$5 \sim 60 \% \mathrm{CH}_{3} \mathrm{CN}$ in $0.1 \% \mathrm{HCOOH}$ in 30 minutes with a flow-rate of $1 \mu \mathrm{l} /$ minute. Samples were ionized with Nanoflow-LC ESI. The LCQ-Deca XP ion trap mass spectrometer (Thermo Electron Corp., USA) was used to obtain MS/MS spectrum data. Phosphorylation was screened with the Mascot database-searching software (Matrix Science, Inc., London).

The mass spectrometry identified tryptic peptides covering $58 \%$ of AfsK $\Delta \mathrm{C}$. Of these peptides, three peptides (precursor ions) were predicted to contain a single phosphoamino acid (Table 1). For example, the Mr of the first peptide in Table 1, predicted from the observed value $\left(\mathrm{Mr}=\right.$ observed $\left.\left[\mathrm{MH}_{2}^{++}\right] \quad(860.58) \times 2-2=1719.16\right)$, is almost the same $($ delta $=0.38)$ as the value $(1718.78)$ calculated on the assumption that a single amino acid is phosphorylated.
Analysis of each precursor ion by MS/MS revealed that Ser-71 for the first peptide in Table 1, Ser-128 for the second, and Thr-168 or Thr-170 for the third were phosphorylated. Figure 1 is the MS/MS spectrum for the third peptide $(\mathrm{m} / z$ 1095.54). For example, peptide ion b7 was observed at $m / z 811.9$, which is very close to the value $(\mathrm{m} / \mathrm{z}$ 811.3) calculated on the assumption that a single amino acid, Thr-168 or Thr-170, is phosphorylated. Consistent with this, peptide ion y12 was observed at $\mathrm{m} / \mathrm{z}$ 1280.5 , which is the value calculated on the assumption that this peptide has no phosphates. Because of the failure to detect $\mathrm{b} 2, \mathrm{~b} 3, \mathrm{y} 17$ or $\mathrm{y} 18$, we could not determine which of the two, Thr-168 or Thr-170, was phosphorylated. As described later, site-directed mutagenesis of the two Thr residues revealed that Thr-168 was actually phosphorylated. 


\section{Site-directed Mutagenesis of Ser-71, Ser-128, Thr-168 and Thr-170}

Of the four amino acid residues, Thr-168 and Thr-170 were presumed to be the functionally important phosphorylated residues because these are located within the activation loop and have been reported to be phosphorylated in PKA [10], PknB [7 9] and several other Ser/Thr kinases. The phosphorylation on Thr residue(s) in AfsK was also consistent with the observation that Thr in AfsK was overwhelmingly phosphorylated, when determined by phosphoamino acid analysis $[5,14]$. No phosphorylation on Ser residues corresponding to position 71 or 128 of Hanks kinases has so far been observed. In addition, these serine residues are not conserved among serine/threonine kinases; for example, the amino acids of PKA and PknB, corresponding to Ser-71, are both Gln and those, corresponding to Ser-128, are Tyr for PKA and Phe for PknB.

We changed the four amino acids as candidates for the functionally autophosphorylated residues of AfsK to Ala by standard site-directed mutagenesis. Correct replacement was checked by direct nucleotide sequencing. These mutant AfsK $\Delta \mathrm{C}$ proteins were similarly produced in $E$. coli, refolded, and incubated with $\left[\gamma_{-}{ }^{32} \mathrm{P}\right]$ ATP to see the effect of the mutations on autophosphorylation. Because a considerable population of the $\operatorname{AfsK} \Delta \mathrm{C}$ sample purified from $E$. coli has been phosphorylated intermolecularly, it phosphorylates non-phosphorylated AfsK in the sample, giving a distinct ${ }^{32} \mathrm{P}$-labeled signal on incubation with $\left[\gamma_{-}{ }^{32} \mathrm{P}\right]$ ATP $[14]$ (see below). As expected, mutant S128A autophosphorylated to the same extent as AfsK $\Delta \mathrm{C}$ and phosphorylated AfsR (data not shown). However, mutant S71A showed no autophosphorylation (data not shown). We assume that the failure of mutant S71A to autophosphorylate is due to an unfavorable conformational change caused by the amino acid replacement or to insufficient refolding. This idea was supported by the observation that a single amino acid replacement at Thr-168 almost completely abolished the ability of AfsK $\Delta \mathrm{C}$ to autophosphorylate (see below).

Thr-168 is conserved in all the Hanks kinases from prokaryotes to eukaryotes, and $\mathrm{Thr}-170$ is also conserved in most of these kinases (Fig. 2A), which implies the biological importance of these threonines, especially Thr168. Mutant T170A autophosphorylated and phosphorylated AfsR, as did AfsK $\Delta C$ (Fig. 3A), which excluded the possibility that Thr-170 was a phosphorylated residue. On the other hand, AfsR-phosphorylating activity of mutant T168A was greatly reduced. The slightly remaining activity toward AfsR was presumed to be due to the basal kinase activity of non-phosphorylated $\operatorname{AfsK} \Delta \mathrm{C}$. A faint
A

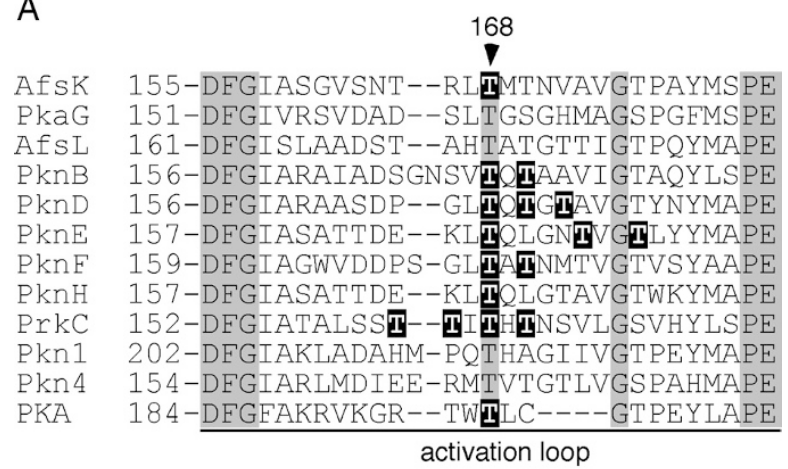

B
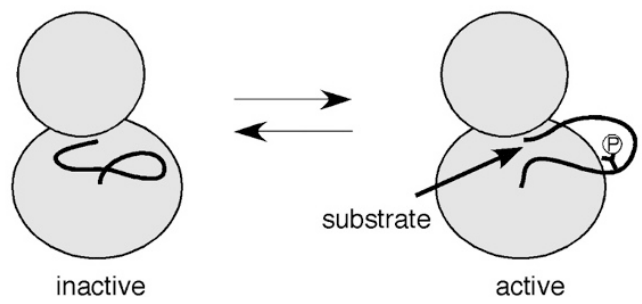

Fig. 2 Alignment of the amino acid sequences forming an activation loop of serine/threonine kinases (A) and schematic illustration of self-activation of kinase activity on phosphorylation of one or more threonine residues within the loop (B).

A. AfsK, PkaG, and AfsL are from Streptomyces; Pkn kinases, from Mycobacterium; PrkC, from Bacillus; Pkn1 and Pkn4, from Myxococcus; and PKA, from Mus musculus. Highly conserved amino acids are shaded. The blackened Thr residues are those experimentally determined to be phosphorylated. B. An illustration for activation of kinase activity on phosphorylation of Thr residue(s) in the activation loop. The non-phosphorylated activation loop on the bi-lobed kinase domain prevents the substrate from access to the catalytic center, whereas the phosphorylation of the loop perturbs the conformation so that the substrate makes an easy access. Modified from Huse and Kuriyan [21].

phosphorylation of AfsK $\Delta \mathrm{C}$ itself was perhaps due to non-specific phosphorylation by the basal kinase activity. Consistent with this idea, AfsK $\Delta \mathrm{C}$ and mutant T170A gave smeared protein bands on SDS-polyacrylamide gel electrophoresis, whereas mutants at position 168 , such as T168A, T168D, or T168E, gave a distinct single band (Fig. 3C). The smeared bands of AfsK $\Delta \mathrm{C}$ and $T 170 \mathrm{~A}$ represent a mixture of the proteins that have been phosphorylated in E. coli cells and during purification mainly at Thr-168 and slightly at nonspecific positions, as was observed previously [14]. We thus concluded that Thr-168 was a residue that was intermolecularly autophosphorylated. Self-activation of AfsK by phosphorylation of a single Thr residue in the activation loop is the same as for eukaryotic PKA [11, 12], but a contrast with $M$. tuberculosis PknB [8] that self- 
A

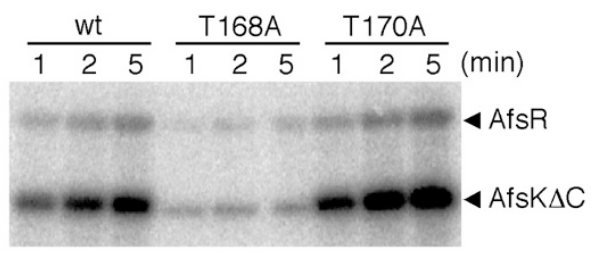

B

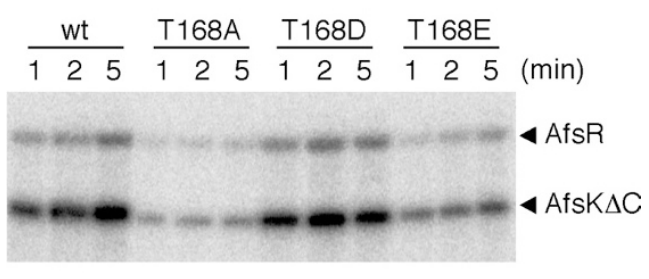

C

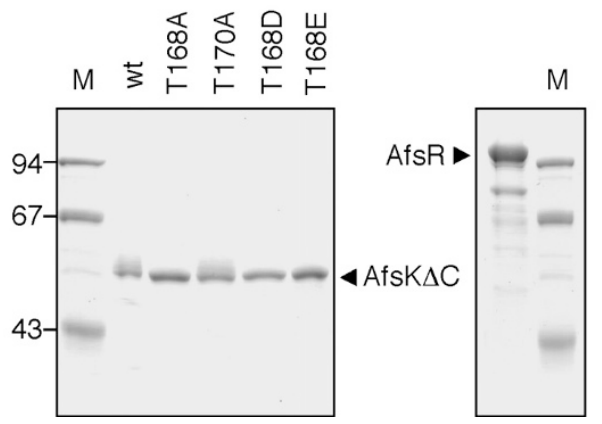

Fig. 3 Autophosphorylation of AfsK $\Delta C$ and site-directed mutant AfsK $\Delta C$ proteins and phosphorylation of AfsR by these proteins (A and $B$ ) and SDS-polyacrylamide gel electrophoresis of AfsK $\Delta C, \operatorname{AfsK} \Delta \mathrm{C}$ mutants and AfsR (C).

$A$ and B. For autophosphorylation of $\operatorname{AfsK} \Delta C$ (wt) and its mutants and phosphorylation of AfsR by these kinases, $30 \mathrm{pmol}$ of AfsR was added to the standard reaction for AfsK $\Delta C$ autophosphorylation, described in the text, and the reaction mixture was incubated at $30^{\circ} \mathrm{C}$ for the indicated minutes. C. AfsK $\Delta C$ and mutant proteins were produced in $E$. coli, refolded, purified, and subjected to SDS-polyacrylamide gel electrophoresis. AfsK $\Delta C$ and mutant T170A give a smeared band. AfsR (993 amino acid residues with a His-tag at its $\mathrm{N}$-terminal end), used as a substrate of AfsK $\Delta C$, was also produced by $E$. coli and similarly analyzed. The molecular size standards used are phosphorylase b (97 kDa), bovine serum albumin (67 kDa), and ovalbumin (43 kDa).

activates by phosphorylation of two Thr residues (Fig. 2A).

Despite sequence identity less than $27 \%$, prokaryotic and eukaryotic serine/threonine kinases show close conformational similarity $[7,11,17]$. The conformational changes in activation of these kinases with a characteristic two-lobed structure are linked to changes at regulatory sites that include phospho-acceptors in the activation loop (Fig. 2B). On phosphorylation of one or more Thr residues in the activation loop, the conformation of the region for the ATP- and substrate-binding sites, including the activation loop, is changed so that the substrate is allowed to make an easy access. The phosphorylation of Thr-168 in AfsK is supposed to cause a similar conformational change, resulting in activation of the kinase activity.

\section{Mutant T168D as a Constitutively Active Kinase}

An amino acid replacement of phosphorylatable Thr and Ser residues by a negatively charged amino acid, Glu or Asp, can mimic the effect of the phosphate group in some eukaryotic Ser/Thr kinases and therefore results in generating constitutively active enzymes [13, 18], although no such examples for prokaryotic Hanks kinases have been shown. The functional importance of phosphorylation at Thr-168 was confirmed by a constitutively active kinase activity of mutant T168D (Fig. 3B). Mutant T168D phosphorylated AfsR to almost the same extent as AfsK $\Delta \mathrm{C}$, indicating that $\mathrm{T} 168 \mathrm{D}$ was a constitutively active enzyme. A distinct phosphorylation of $\operatorname{AfsK} \Delta \mathrm{C}$ itself was presumed to be due to non-specific phosphorylation. Mutant T168E was less active than mutant T168D but more active than T168A. We thus assume that intermolecular phosphorylation of Thr-168 accounts for the self-activation of AfsK $\Delta \mathrm{C}$ and that the remaining $\mathrm{C}$-terminal portion of AfsK, probably interacting with other proteins [19], causes no effects on autophosphorylation even of full-length AfsK.

\section{The AfsK-AfsR-AfsS System for Regulation of Secondary Metabolism}

The AfsK-AfsR signal transduction pathway so far demonstrated or suggested is as follows. AfsK loosely attached to the inner side of the membrane autophosphorylates a single threonine residue at position 168 , perhaps sensing some external or internal signal, and enhances its own kinase activity. The kinase activity is modulated by KbpA, an AfsK-binding protein, by means of protein-protein interaction [14]. The activated AfsK phosphorylates AfsR in the cytoplasm, by which the DNAbinding activity of AfsR is greatly enhanced [15]. In addition to AfsK, other kinases, including $\mathrm{PkaG}$ and $\mathrm{AfsL}$ (Fig. 2A), also phosphorylate AfsR, suggesting that AfsR serves as an integrator of multiple signals sensed by these kinases $[2,16]$. Because of high end-to-end similarity of these kinases to AfsK, they are supposed to self-activate by autophosphorylation at a single Thr residue corresponding to Thr-168. The phosphorylated AfsR binds the promoter of $a f s S$, which encodes a protein of 63 amino acids, and forms a closed complex with RNA polymerase [15]. The closed complex is then converted to a transcriptionally active open complex, perhaps by the energy available from ATP hydrolysis by AfsR. AfsS induced in this way 
activates transcription of pathway-specific activators, such as actII-ORF4 for actinorhodin production and redD for undecylprodigiosin [15], in an as yet unknown manner. The AfsK/AfsR system forming a possible regulatory network with other AfsK-type kinases, which is conserved widely among Streptomyces, is supposed to play a regulatory role for secondary metabolism and morphological differentiation. In fact, the AfsK/AfsR system affects aerial mycelium formation in $S$. griseus [20].

Acknowledgments We are grateful to the support of BSI's Research Resources Center for the MS analysis. This work was supported by a Grant-in-Aid for Scientific Research on Priority Areas from Monkasho and the BioDesign Program of the Ministry of Agriculture, Forestry, and Fisheries of Japan.

References

1. Umeyama T, Lee P-C, Horinouchi S. Protein serine/ threonine kinases in signal transduction for secondary metabolism and morphogenesis in Streptomyces. Appl Microbiol Biotechnol 59: 419-425 (2002)

2. Horinouchi S. AfsR as an integrator of signals that are sensed by multiple serine/threonine kinases in Streptomyces coelicolor A3(2). J Ind Microbiol Biotechnol 30: 462-467 (2003)

3. Bentley SD, Chater KF, Cerdeño-Tarraga AM, Challis GL, Thomson NR, James KD, Harris DE, Quail MA, Kieser H, Harper D, Bateman A, Brown S, Chandra G, Chen CW, Collins M, Cronin A, Fraser A, Goble A, Hidalgo J, Hornsby T, Howarth S, Huang CH, Kieser T, Larke L, Murphy L, Oliver K, O’Neil S, Rabbinowitsch E, Rajandream MA, Rutherford K, Rutter S, Seeger K, Saunders D, Sharp S, Squares R, Taylor K, Warren T, Wietzorrek A, Woodward J, Barrell BG, Parkhill J, Hopwood DA. Complete genome sequence of the model actinomycete Streptomyces coelicolor A3(2). Nature 417: 141-147 (2002)

4. Ikeda H, Ishikawa J, Hanamoto A, Shinose M, Kikuchi H, Shiba T, Sakaki Y, Hattori M, Ōmura S. Complete genome sequence and comparative analysis of the industrial microorganism Streptomyces avermitilis. Nat Biotechnol 21: 526-531 (2003)

5. Matsumoto A, Hong S-K, Ishizuka H, Horinouchi S, Beppu T. Phosphorylation of the AfsR protein involved in secondary metabolism in Streptomyces species by a eukaryotic-type kinase. Gene 146: 47-56 (1994)

6. Madec E, Stensballe A, Kjellström S, Cladière L, Obuchowski M, Jensen ON, Séror SJ. Mass spectrometry and site-directed mutagenesis identify several autophosphorylated residues required for the activity of PrkC, a Ser/Thr kinase from Bacillus subtilis. J Mol Biol 330: 459-472 (2003)
7. Young TA, Delagoutte B, Endrizzi JA, Falick AM, Alber T. Structure of Mycobacterium tuberculosis PknB supports a universal activation mechanism for Ser/Thr protein kinases. Nat Struct Biol 10: 168-174 (2003)

8. Boitel B, Ortiz-Lombardia M, Durán R, Pompeo F, Cole ST, Cerveñansky $\mathrm{C}$, Alzari PM. PknB kinase activity is regulated by phosphorylation in two $\mathrm{Thr}$ residues and dephosphorylation by PstP, the cognate phospho-Ser/Thr phosphatase, in Mycobacterium tuberculosis. Mol Microbiol 49: 1493-1508 (2003)

9. Durán R, Villarino A, Bellinzoni M, Wehenkel A, Fernandez P, Boitel B, Cole ST, Alzari PM, Cerveñansky C. Conserved autophosphorylation pattern in activation loops and juxtamembrane regions of Mycobacterium tuberculosis Ser/Thr protein kinases. Biochem Biophys Res Commun 333: 858-867 (2005)

10. Molle V, Kremer L, Girard-Blanc C, Besra GS, Cozzone AJ, Prost J-F. An FHA phosphoproteins recognition domain mediates protein EmbR phosphorylation by $\mathrm{PknH}$, a Ser/Thr protein kinase from Mycobacterium tuberculosis. Biochemistry 42: 15300-15309 (2003)

11. Batkin M, Schvartz I, Shaltiel S. Snapping of the carboxyl terminal tail of the catalytic subunit of PKA onto its core: characterization of the sites by mutagenesis. Biochemistry 39: 5366-5373 (2000)

12. Akamine P, Madhusudan, Wu J, Xuong NH, Ten Eyck LF, Taylor SS. Dynamic features of cAMP-dependent protein kinase revealed by apoenzyme crystal structure. J Mol Biol 327: 159-171 (2003)

13. Stein SC, Woods A, Jones NA, Davison MD, Carling D. The regulation of AMP-activated protein kinase by phosphorylation. Biochem J 345: 437-443 (2000)

14. Umeyama T, Horinouchi S. Autophosphorylation of a bacterial serine/threonine kinase, AfsK, is inhibited by KbpA, an AfsK-binding protein. J Bacteriol 183: 5506-5512 (2001)

15. Lee P-C, Umeyama T, Horinouchi S. afs $S$ is a target of AfsR, a transcriptional factor with ATPase activity that globally controls secondary metabolism in Streptomyces coelicolor A3(2). Mol Microbiol 43: 1413-1430 (2002)

16. Sawai R, Suzuki A, Takano Y, Lee P-C, Horinouchi S. Phosphorylation of AfsR by multiple serine/threonine kinases in Streptomyces coelicolor A3(2). Gene 334: 53-61 (2004)

17. Ortiz-Lombardía M, Pompeo F, Boitel B, Alzari PM. Crystal structure of the catalytic domain of the $\mathrm{PknB}$ serine/threonine kinase from Mycobacterium tuberculosis. J Biol Chem 278: 13094-13100 (2003)

18. Cowley S, Paterson H, Kemp P, Marshall CJ. Activation of MAP kinase is necessary and sufficient for PC12 differentiation and for transformation of NIH $3 \mathrm{~T} 3$ cells. Cell 77: 841-852 (1994)

19. Neu JM, MacMillan SV, Nodwell JR, Wright GD. StoPK-1, a serine/threonine protein kinase from the glycopeptide antibiotic producer Streptomyces toyocaensis NRRL 15009, 
affects oxidative stress response. Mol Microbiol 44: 417-430 (2002)

20. Umeyama T, Lee P-C, Ueda K, Horinouchi S. An AfsK/AfsR system involved in the response of aerial mycelium formation to glucose in Streptomyces griseus. Microbiology 145: 2281-2292 (1999)

21. Huse M, Kuriyan J. The conformational plasticity of protein kinases. Cell 109: 275-282 (2002) 